\title{
A stochastic MCMC elastic waveform inversion method for multicomponent data
}

Allan Peixoto de Franco LENEP/UENF

Sérgio Adriano Moura Oliveira LENEP/UENF, INCT-GP and Invision Geophysics

Fernando Sérgio de Moraes LENEP/UENF, INCT-GP and Invision Geophysics

Copyright 2021, SBGf - Sociedade Brasileira de Geofísica

This paper was prepared for presentation during the $17^{\text {th }}$ International Congress of the Brazilian Geophysical Society held in Rio de Janeiro, Brazil, 16-19 August 2021.

Contents of this paper were reviewed by the Technical Committee of the $17^{\text {th }}$ International Congress of the Brazilian Geophysical Society and do not necessarily represent any position of the SBGf, its officers or members. Electronic reproduction or storage of any part of this paper for commercial purposes without the written consent of the Brazilian Geophysical Society is prohibited.

\begin{abstract}
Most of the inversion done to infer the elastic properties of the subsurface rock layers, in the oil E\&P industry, continues to rely on conventional seismic data that contains PP waves only, despite the benefits that the joint PP-PS data may bring. Here we present a stochastic Bayesian seismic waveform inversion method for multicomponent data, accounting for the full elastic response of the layered media. The proposed inversion routine evaluates $V p, V s, \rho$ and its associated uncertainties using the Monte Carlo Markov Chain method to sampling the posterior distribution. We present examples involving synthetic and also real data. These tests showed a great reduction in the uncertainties associated with the estimation of Vs using the PP-PS data, compared with the PP data.
\end{abstract}

\section{Introduction}

Seismic inversion aims on performing quantitative estimations of the elastic parameters of subsurface rocks. Elastic inversion became an essential routine tool especially employed in exploration and reservoir characterization for the E\&P industry. Its origin can be traced back in the 1980s, with pioneering works on the development of AVO (amplitude versus offset) technology, published by Ostrander (1984), Sherwood et al. (1983), Shuey (1985) e Smith \& Gidlow (1987). These early developments rely only on primaries waves ( $P$ waves), but the development of multicomponent seismic in the 1990s enabled the recording of converted PS waves and led to the emergence of joint PP-PS elastic inversion schemes, see e.g. Stewart (1990). Several studies claim that joint PP-PS inversion produces superior results when compared to conventional PP inversion, especially for density and S-wave impedance (see e.g., Jin 1999, Mallick 2000, Mahmoudian and Margrave 2003), however higher costs associated with the acquisition and processing of multicomponent seismic data has made PP-PS inversion much less used then conventional PP inversion until now.

Seismic data are band limited, have a limited angle coverage and is contaminated by noise. As a result, seismic inversion is an ill posed problem, once the data may be explained by many different models within the error bounds. The linear inversion approach offers an elegant and convenient way to analyze these effects in elastic inversion, since a covariance or resolvability matrix can be readily obtained by means, for example, of SVD technique; (De Haas and Berkhout, 1988), (Rijssen and Herman, 1991), (Jing and Rape, 2004), (Khare and Rape, 2007). Another way to deal with ill-posed inverse problems is to use stochastic methods that perform an extensive random or pseudo random exploration of the model space to test many possible solutions (Sambridge and Mosegaard 2002). In stochastic inversion, the model parameters are treated as random variables and the solution is given by a probability density function (Tarantola, 1987). The advantages of this approach are that it is not necessary to assume a linear relation between data and model parameters and the possibility of calculation of uncertainty associated with the estimates (Gouveia and Scales, 1997, 1998).

The practical elastic inversion method commonly used in the E\&P industry assume the subsurface to be locally 1-D and is based on convolutional model that uses approximate formulas for the reflection coefficients in order to explain the amplitudes of the pre-stack seismic data. This allows for the linearization of the inverse problem and facilitates the calculation of uncertainty, since a closed form of the posterior probability function is available (Buland and Omre, 2003). However, these approximated formulas assume small contrasts in elastic properties between layers and are not valid for post critical angles of incidence. Besides this, the linearized elastic inversion also assumes that the seismic response of the earth contains only primary reflections. This simplified inversion scheme is not able to correctly explain the amplitudes related to high incidence angles, especially for thin layers with high contrast between the elastic properties (Simons and Backus, 1994; Mallick, 2007).

The heart of the proposed stochastic estimation method is of an efficient modelling procedure used to calculate prestack PP and PS angle gathers using the reflectivity method, that accounts for the full seismic response of the layered media. The inversion is parametrized in terms of the primary wave velocity $V_{p}$, secondary wave velocity $V_{s}$ and density $\rho$ and assumes Gaussian prior statistics about the medium elastic parameters. The Metropolis algorithm from Monte Carlo Markov Chain methods is used to sampling the posterior distribution and estimators like mean and uncertainties like standard deviations are obtained from these sample values. 


\section{Modelling of PP and PS angles gathers}

In this work we consider the upgoing $P$ waves and $S$ waves that are generated by the incidence of a down going transient elastic plane $P$ wave at different angles on an elastic layered medium. These waves will be referred here as PP and PS waves. We consider the layers to be isotropic and homogeneous. It is important that the calculation of the seismic response takes into account all events generated in the layer, like multiples, transmission effects and mode conversions of all types and not only primaries reflections. This is due to the influence of these wave modes on the amplitude of the recorded signal. This influence becomes more evident with the decrease of the width of the layer and with the increase of the contrast and incidence angle, as noted by Simmons and Backus (1994).

The reflectivity method (Muller, 1985) is used for modeling the seismic response of the layered medium. The PP and PS plane wave responses can be given as a function of the ray parameter $p=\sin \theta / \alpha_{0}$ and the vertical two way travel time $\tau$ by the following inverse Fourier transforms:

$$
\begin{aligned}
& s_{p p}(\tau, p)=\frac{1}{2 \pi} \int_{-\infty}^{\infty} r_{p p}^{0}(\omega) e^{-i 2 k_{z}^{0} z_{1}} F(\omega) e^{-i \omega \tau} d \omega \\
& s_{p s}(\tau, p)=\frac{1}{2 \pi} \int_{-\infty}^{\infty} r_{p s}^{0}(\omega) e^{-i\left(k_{z}^{0}+\eta_{z}^{0}\right) z_{1}} F(\omega) e^{-i \omega \tau} d \omega
\end{aligned}
$$

Where $z_{1}$ is the depth of the top interface, $\alpha_{0}$ and $\beta_{0}$ are respectively the $P$ wave and $S$ wave velocity of the upper semi space. The frequency domain function of the seismic pulse is $F(\omega), r_{p p}^{0}$ is the PP reflectivity and $r_{p s}^{0}$ is the PS reflectivity. Those are the elements of the reflectivity matrix. The vertical wave numbers for $\mathrm{P}$ and $\mathrm{S}$ waves in the uper semispace are, respectively; $k_{z}^{0}=\sqrt{\left(\frac{1}{0}\right)^{2} p^{2}}$ and ${ }_{z}^{0}=\sqrt{\left(\frac{1}{0}\right)^{2} \quad p^{2}}$.

In practical applications, the input seismic data for prestack inversion is sorted by offset or by incidence angle. Due to this, the PP and PS plane wave seismograms are submitted to tau-p NMO correction (Diebold and Stoffa, 1981) and then transformed from ray parameter domain to incidence angle domain by means of a simple mapping procedure, applied along the two way travel time coordinate, based on Snell low for elastic waves: $p=\sin { }_{p p} /{ }_{0}=\sin { }_{p s} /{ }_{0}$, where $\theta_{p p}=\theta$ is the PP reflection angle and $\theta_{p s}$ is the PS conversion angle. This kind of data is known as angle gather seismogram.

\section{Bayesian Inversion by MCMC}

The goal of the Bayesian inversion is to obtain the probability density function that describes the parameters of the medium. This is known as the posterior probability distribution and is given by the formula

$$
P(\mathbf{m} \mid \mathbf{d})=k P(\mathbf{d} \mid \mathbf{m}) P(\mathbf{m})
$$

Where $P(\mathbf{d} \mid \mathbf{m})$ is the likelihood distribution probability function of the observed data $\mathbf{d}$ given a model $\mathbf{m}, \mathbf{P}(\mathbf{m})$ is the prior probability density function of $\mathrm{m}$ and $k$ is a normalization constant. We assume that the data error is approximately Gaussian distributed, implying that the likelihood function can be written as

$$
P(\mathbf{d} \mid \mathbf{m})=\exp \left[\frac{1}{2}(\mathbf{d} \quad G(\mathbf{m}))^{T}\left(\mathbf{C}_{e}\right){ }^{1}(\mathbf{d} \quad G(\mathbf{m}))\right]
$$

in which $\mathbf{d}$ is the observed data vector (that can be PP or PP-PS data), $\mathbf{m}$ is the vector of model parameters, $G($ ) is the forward modeling operator and $\mathbf{C}_{e}$ is the covariance matrix that, in our case, is a simple diagonal matrix with a constant value for the data variances.

We use the classical Metropolis algorithm (Metropolis et al., 1953; Chib and Greenberg, 1995) to sample the posterior distribution. This method can be summarized by two steps: the first is the exploration step, when a proposed model $\mathbf{m}_{\mathrm{p}}$ is drawn from a current model $\mathbf{m}_{\mathrm{c}}$ using a proposal distribution. In the second step this proposed model is accepted or rejected according to the following rule:

$$
\begin{aligned}
& \text { - Accept } \mathbf{m}_{p} \text {, if } P\left(\mathbf{d} \mid \mathbf{m}_{p}\right) \geq P\left(\mathbf{d} \mid \mathbf{m}_{c}\right) . \\
& \text { - If } P\left(\mathbf{d} \mid \mathbf{m}_{p}\right)<P\left(\mathbf{d} \mid \mathbf{m}_{c}\right) \text {, accept } \mathbf{m}_{p}, \\
& \text { with probability } \frac{P\left(\mathbf{d} \mid \mathbf{m}_{p}\right)}{P\left(\mathbf{d} \mid \mathbf{m}_{c}\right)} \text {. }
\end{aligned}
$$

It can be shown that after a certain number of iterations, called the burn-in period, the statistics of the accepted models set will reach an equilibrium, converging to the posterior distribution.

\section{Test on synthetic data}

In this section we test the elastic inversion involving PP and PS data in a layered model. This model contains seven layers with variable thickness and contrasts. The PP and PP-PS inversions are performed considering $0-40^{\circ}$ angle range. The results are shown in Figure 1. Here we calculate only the mean and standard deviation for each one of the 21 parameters. For the prior distribution we choose a box function that assigns a constant prior probability in a search window of $\pm 30 \%$ around the real value of each parameter. We use the Metropolis algorithm with six independent runs of 5000 samples each, discarding 2000 due to the burn in period and keeping the last 3000 samples to derive the statistics of the posterior distribution. 
By comparing the PP and PP-PS inversions, we note that the inclusion of the PS data in the PP-PS inversion improves $V_{s}$ estimates. The density estimation proved to be especially difficult and this is true for all PP and PP-PS inversions. In Figure 2, we show the histograms of the estimated parameters with $0-40^{\circ}$ input angle range. We note in these figures that while the $V_{p}$ and $V_{s}$ histograms are well behaved, the density histograms are characterized by a platykurtic multimodal distribution, where the mean value may be not close to the true value of the parameter. This fact was also noted, for the PP case, by Aleardi and Mazzotti (2017), and we see that this problem still persists in the joint PP-PS inversion.
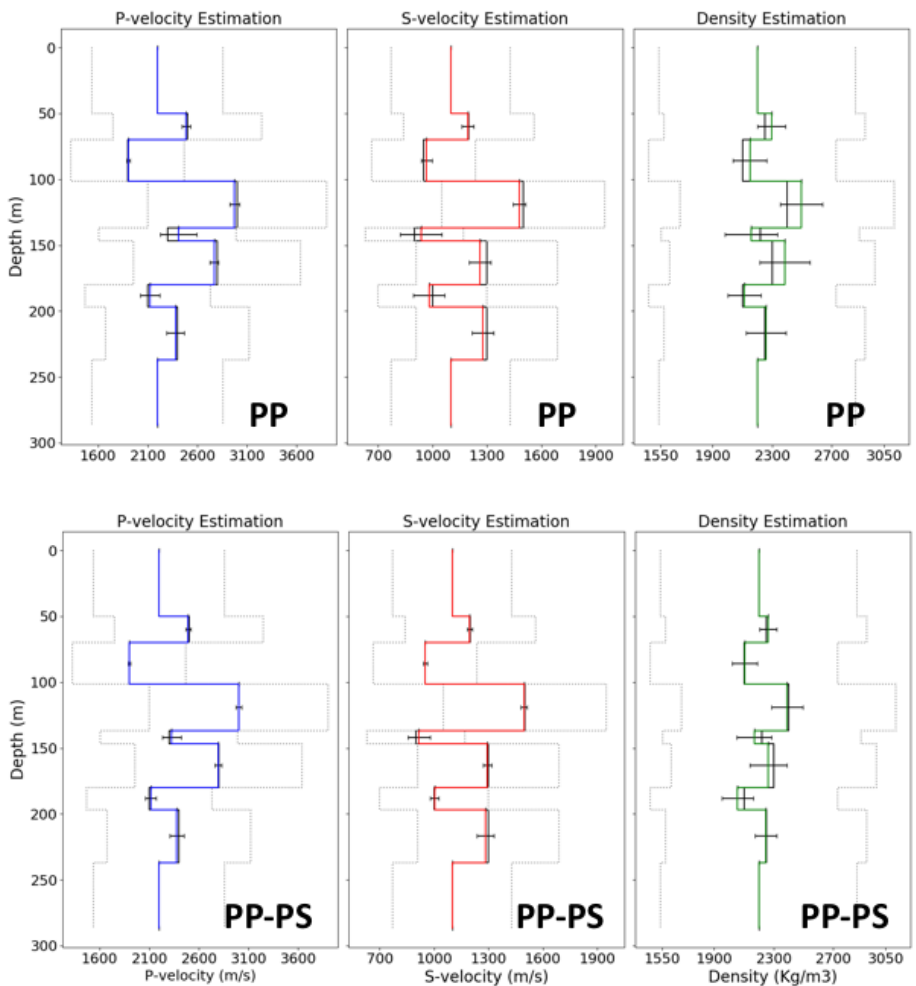

Figure 1 - Inversion results for synthetic data. The blue, red and green lines correspond to the estimates of $V_{p}, V_{s}$ and $\rho$ respectively. The standard deviations of the estimates are represented by the horizontal bars. The gray lines represent the intervals where the prior distribution is different from zero.

\section{Test on real data}

We applied the proposed stochastic inversion approach to a multicomponent data acquired with ocean bottom sensors in an oil field in the Campos basin, RJ, Brazil. It consists of PP and PS image gathers obtained by prestack time migration. This data is noisy (specially the PS gathers) and had to go through a conditioning step, which included multispectral curvelet filtering, inverse $Q$ filtering, residual moveout correction and residual multiple suppression (Franco et al., 2015). The PP and PS image gathers where then transformed to the angle domain using an angle stacking routine that used smooth velocity models extracted from well information and velocity analysis in order to trace rays and then relate offset with incidence angle. The obtained angle stacks contain eight traces that covers angle from 4 to 32 degrees with regular interval of 4 degree (Figures $5 a$ and $5 c$ ). Two seismic pulses were then statistically estimated; one from the PP data and other from the PS data.
The first inversion test was performed using a box like prior distribution $P(m)$ that assigns a uniform probability to a range of values around an average value and zero probability for parameters values outside this range;

$$
P(m)=\left\{\begin{array}{ccc}
\frac{1}{m} \text { se } \mid m & \bar{m} \mid \leq{ }_{m} \\
0 & \text { se } \mid m & \bar{m} \mid>{ }_{m}
\end{array}\right.
$$

The average values $\bar{m}$ was obtained by applying a moving window operator in the well logging curves. This distribution is not very informative about the behavior of the parameters, what make the estimation more dependent on the data than on the prior knowledge. For the stochastic inversion we used six independent runs of 10000 samples each, discarding 2500 due to the burn in period and keeping the last 7500 samples to derive the statistics of the 
posterior distribution. The results of this inversion are shown in Figure $3 \mathrm{a}$. We choose as estimators the mean value of the set of $V_{p}, V_{s}$ and $\rho$ samples generated by the Metropolis algorithm. These samples values are also plotted (in light gray) for each elementary layer. The extent that these samples are dispersed around the mean value gives a clear idea of the uncertainty associated with the estimate of the parameter in question. We see that the estimator for $V_{p}$ are in good agreement with the curve measured from well (filtered to the seismic bandwidth) and the uncertainty associated with this parameter is relatively small. The estimator for $\mathrm{V}_{\mathrm{s}}$ has also a good agreement with the well measurement. The density is the worst resolved parameter and the uncertainty associated with this parameter is much bigger than that of $\mathrm{V}_{\mathrm{p}}$ and $\mathrm{V}_{\mathrm{s}}$. Note that this result, in general, confirms what was predicted with the test on synthetic data (Figure 1).

\section{(a)}
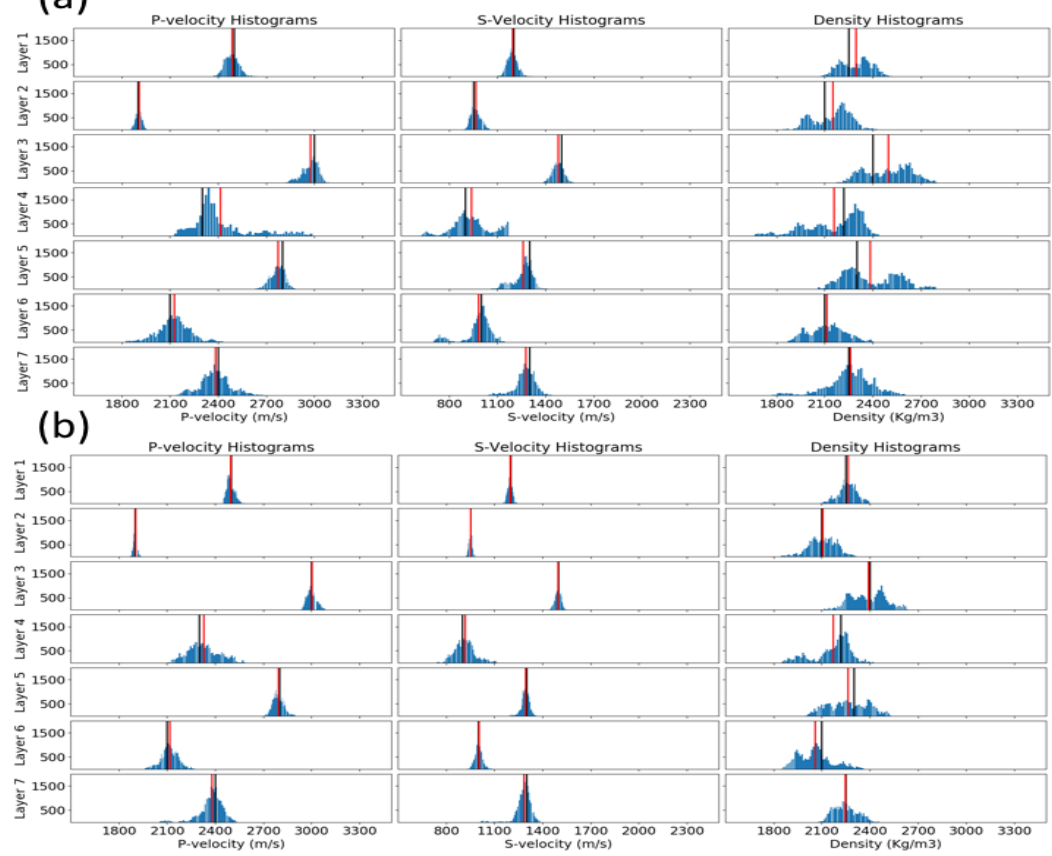

Figue 2 - Histograms associated with estimates of $V_{p}, V_{s}$ and $\rho$ using synthetic data. The vertical red lines indicate the mean of the estimates, the vertical black line represents the reference value of the parameter.

In the second test we use a Gaussian prior distribution for $P(m)$. This distribution is much more informative about the parameters then the box like prior and is given by the following formula:

$$
P(\mathbf{m})=\exp \left[\frac{1}{2}(\mathbf{m} \quad)^{T}\left(\mathbf{C}_{m}\right)^{1}(\mathbf{m} \quad)\right]
$$

Where $\mu$ is the mean vector and $\mathbf{C}_{\mathbf{m}}$ is the matrix whose elements are the covariances of the parameters. In this example, the covariances were obtained from the measurements of $V_{p}, V_{s}$ and $\rho$ made along the well, by using a moving window. For simplification we considered that the covariance of parameters that lies at different elemental layers are zero, so $\mathbf{C}_{\mathbf{m}}$ becomes a block diagonal matrix. We see that the introduction of the Gaussian prior distribution improved significantly the estimates of $V_{s}$ and $V_{p}$ (Figure $3 b$ ). Some improvement in the estimative of density is also present, since there was a decrease in the uncertainty of this parameter, however the estimator for density continues not matching well with the measurements. We see in Figure 4 that the modelled PP and PS angle gathers obtained using the estimates made with the Gaussian prior matches very well with the real angle gathers. 

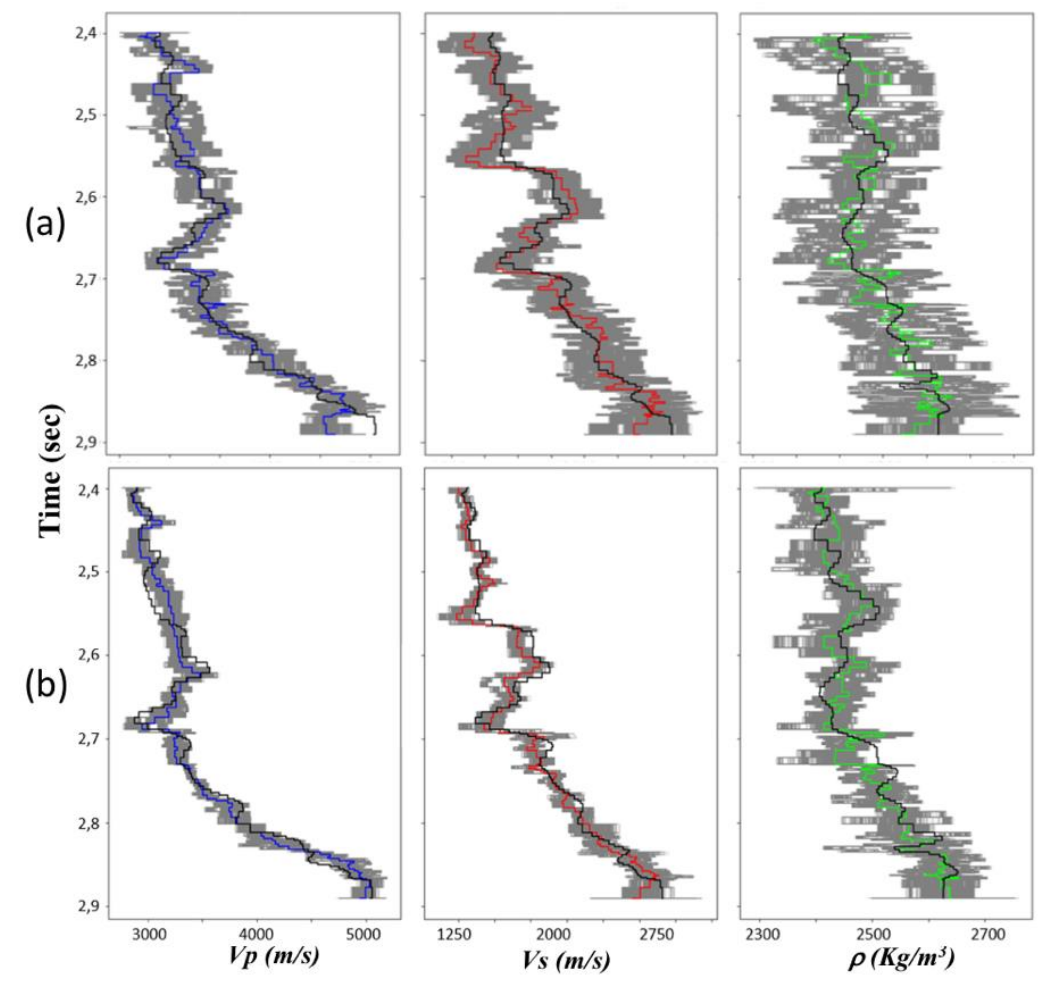

Figure 3 - Real data examples: results of stochastic inversion with (a) uniform and (b) Gaussian priori distribution. The blue, red and green lines correspond to the estimates (mean) of $V_{p}, V_{s}$ and $\rho$ respectively. The samples values generated by the Metropolis algorithm are plotted in light gray. The measured curves from well login are plotted in black.

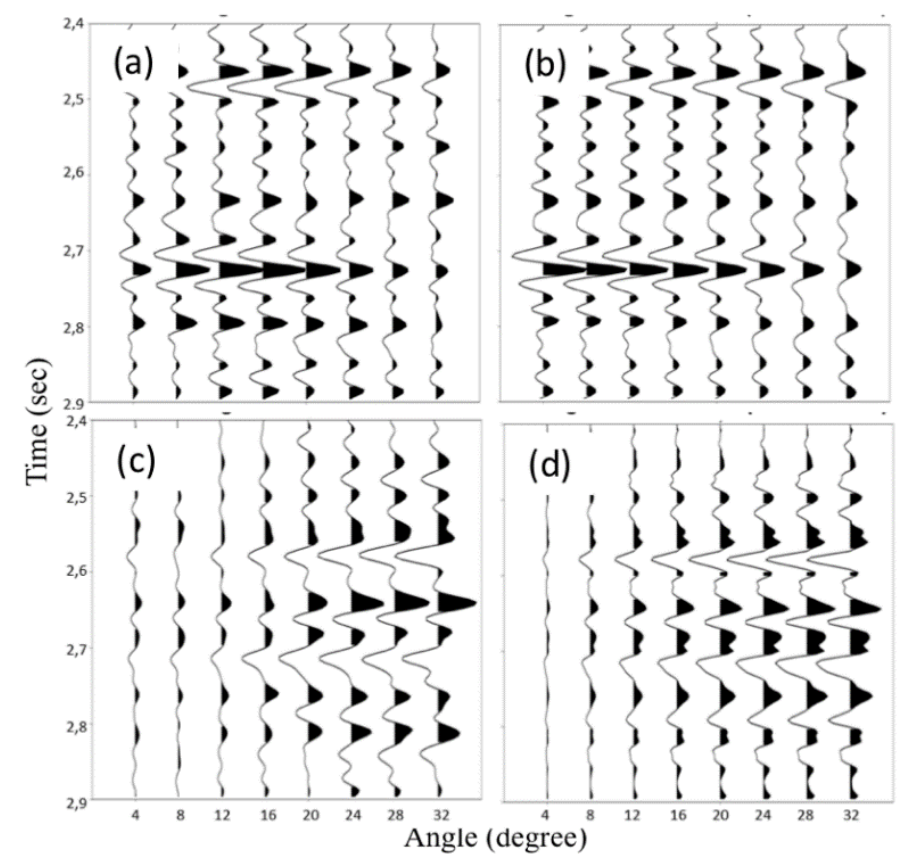

Figure 4 - (a) The real PP angle gather (b) the modelled PP angle gather (c) The real PS angle gather and (d) the modelled $P S$ angle gather obtained using the estimates shown in figure 3 . 


\section{Conclusions}

In this work we develop and present a Bayesian stochastic inversion method for multicomponent seismic data whose main characteristic is the use of an optimized routine for modeling PP and PS angle gathers based on the reflectivity method. The application of such a method in synthetic and real data demonstrated that the parameters $V_{p}$ and $V_{s}$ can be robustly estimated, even from multicomponent data with limited incidence angle coverage. The density estimate was not very robust, and the use of an informative prior distribution is fundamental to reduce the uncertainties associated with this parameter.

\section{Acknowledgments}

We thank PETROBRAS for financial support.

\section{References}

ALEARDI M. \& MAZZOTTI A. 2017. 1D elastic fullwaveform inversion and uncertainty estimation by means of a hybrid genetic algorithm-Gibbs sampler approach: Geophysical Prospecting, 65, no. 1, 64-85.

BULAND A. \& OMRE H. 2003. Bayesian linearized AVO inversion. Geophysics, 68, 185-198

CHIB S. \& GREENBERG E. 1995. Understanding the Metropolis-Hastings algorithm: The American Statistician, JSTOR, 49(4), 327-335.

DE HASS JC. \& BERKHOUT AJ. 1988. On the Information Content of P-P, P-SV, SV-SV, and SV-P Reflections: 58th Annual International Meeting, SEG., Expanded Abstracts, 1190-1194, doi: 10.1190/1.1892487.

DIEBOLD JB. \& STOFFA PL. 1981. The travel time equation, tau-p mapping and inversion of common midpoint data: Geophysics, 46, 238-254, doi: 10.1190/1.1441196.

GOUVEIA W. \& SCALES JA. 1997. Resolution in seismic waveform inversion: Bayes vs. Occam: Inverse Problems, 13, 323-349, doi: 10.1088/02665611/13/2/009.

GOUVEIA W. \& SCALES JA. 1998. Bayesian seismic waveform inversion: Parameter estimation and uncertainty analysis: Journal of Geophysical Research, 103, 2759-2779, doi: 10.1029/97JB02933.

JIN S. 1999. Characterizing reservoir by using jointly Pand S- wave AVO analyses: 69th Annual International Meeting, SEG Expanded Abstracts, 687-690, doi: 10.1190/1.1821117.

JING C. \& RAPE T. 2004. Resolvability analysis of rock property inversions of multicomponent seismic data: 74th Annual International Meeting, SEG, Expanded Abstracts, 897-900, doi: 10.1190/1.1845313.

KHARE V. \& RAPE T. 2007. Density inversion using joint PP/PS data: sensitivity to the angle range: 77th Annual
International Meeting, SEG Expanded Abstracts, 965969, doi: 10.1190/1.2792566.

MALLICK S. 2000. Prestack waveform inversion of multicomponent seismic data: SEG Expanded Abstracts, 19, 2273-2276, doi: 10.1190/1.1815910.

MALLICK S. 2007. Amplitude-variation-with-offset, elasticimpedance, and wave-equation synthetics - $\mathrm{A}$ modeling study: Geophysics, 72, C1-C7, doi: 10.1190/1.2387108.

MAHMOUDIAN F. \& MARGRAVE GF. 2003. AVO inversion of multicomponent data for $P$ and $S$ impedance: CREWES Research Report, 15, 1-34.

MÜLLER G. 1985. The reflectivity method: A tutorial: Journal of Geophysical Research, 58, 153-174.

METROPOLIS N., ROSENBLUTH AW., ROSENBLUTH MN., TELLER AH., \& TELLER E. 1953. Equation of state calculations by fast computing machines: The Journal of Chemical Physics, 21, 1087-1092, doi: 10.1063/1.1699114.

OSTRANDER, WJ.1984. Plane-Wave Reflection Coefficients for Gas Sands at Non-Normal Angles of Incidence: Geophysics, 49, 1637-1648, doi: 10.1190/1.1441571.

RIJSSEN EPF. \& HERMAN GC. 1991. Resolution analysis of band-limited and offset-limited seismic data for plane-layered subsurface. Geophysical Prospecting, Wiley, 39: 61-76

SAMBRIDGE M. \& MOSEGAARD K. 2002. Monte Carlo methods in geophysical inverse problems: Reviews of Geophysics, 40, 3-29, doi: 10.1029/2000RG000089.

SHERWOOD JWC., HILTERMAN FJ., NEALE RN. \& CHEN KC. 1983. Synthetic seismograms with offset for a layered elastic medium: SEG Technical Program Expanded Abstracts, 444-447, doi: 10.1190/1.1893660.

SHUEY RT. 1985. A simplification of the Zoeppritz equations: Geophysics, 50, 609-614, doi: 10.1190/1.1441936.

SIMMONS JL. \& BACKUS MM. 1994. AVO modeling and the locally converted shear wave: Geophysics, 59, 1237-1248, doi: 10.1190/1.1443681.

SMITH GC. \& GIDLOW PM. 1987. Weighted stacking for rock property estimation and detection of gas: Geophysical Prospecting, 35, 993-1014, doi: 10.1111/j.1365-2478.1987.tb00856.x.

STEWART RR. 1990. Joint $P$ and P-SV inversion: CREWES Research Report, 2, 112-115.

TARANTOLA A. 1987. Inverse problem theory - methods for data fitting and model parameter estimation: Elsevier Science, eBook ISBN: 9780444599674. 